\title{
Constraining the Evolution of Young Radio-Loud AGN
}

\author{
I. A. G. Snellen ${ }^{1}$, K.-H. Mack ${ }^{2,3,4}$, R. T. Schilizzi ${ }^{5,6}$ and W. Tschager 6 \\ ${ }^{1}$ Institute for Astronomy, University of Edinburgh, Blackford Hill, Edinburgh EH9 3HJ, UK \\ ignas@roe.ac.uk \\ ${ }^{2}$ ASTRON/NFRA, Postbus 2, NL-7990 AA Dwingeloo, The Netherlands \\ ${ }^{3}$ Istituto di Radioastronomia de CNR, Via Gobetti 101, I-40129 Bologna, Italy \\ mack@ira.cnr.it \\ ${ }^{4}$ Radioastronomisches Institut der Universität Bonn, Auf dem Hügel 71, D-53121 Bonn, Germany \\ ${ }^{5}$ Joint Institute for VLBI in Europe, Postbus 2, NL-7990 AA, Dwingeloo, The Netherlands \\ schilizzi@jive.nl \\ ${ }^{6}$ Leiden Observatory, P.O. Box 9513, NL-2300 RA, Leiden, The Netherlands \\ tschager@strw.leidenuniv.nl
}

Received 2002 July 5, accepted 2002 October 15

\begin{abstract}
GPS and CSS radio sources are the objects of choice to investigate the evolution of young radioloud AGN. Previous investigations, mainly based on number counts and source size distributions, indicate that GPS/CSS sources decrease significantly in radio power when evolving into old, extended objects. We suggest this is preceded by a period of increase in radio luminosity, which lasts as long as the radio source is confined within the core-radius of its host galaxy. We have selected a sample of nearby compact radio sources, unbiased by radio spectrum, to determine their luminosity function, size distribution, dynamical ages, and emission line properties in a complete and homogeneous way. First results indicate that the large majority of objects ( $>80 \%$ ) exhibit classical GPS/CSS radio spectra, and show structures consistent with them being compact double or compact symmetric objects. This sample provides an ideal basis to further test and constrain possible evolution scenarios, and to investigate the relation between radio spectra and morphologies, orientation and Doppler boosting in samples of young radio-loud AGN, in an unbiased way.
\end{abstract}

Keywords: galaxies: active — galaxies: jets — radio continuum: galaxies

\section{Current Views on Radio Source Evolution}

It has been shown beyond reasonable doubt that the archetypical gigahertz peaked spectrum (GPS) radio galaxies are young objects, with typical dynamical ages of $10^{2}-10^{3}$ years (Polatidis \& Conway 2003, and references therein). These sources are therefore the prime candidates to be the young progenitors of classical large-size radio sources, which subsequently evolve into compact steep spectrum (CSS), and FR I and/or FR II radio sources. Although it is not clear whether all GPS sources are young objects (certainly the GPS quasars seem to be an unrelated distinct class of objects), GPS galaxies are the key objects to study the early evolution of radio-loud AGN.

Current views on radio source evolution are mainly based on the radio power-linear size $(P-D)$ diagram, and source size distributions. These suggest that if GPS and CSS sources evolve into large-size radio sources, they should decrease in radio power by at least an order of magnitude, to account for their relatively high space density (Fanti et al. 1995; Readhead et al. 1996; O’Dea \& Baum 1997). This luminosity evolution scenario is backed up by theory, i.e. it is expected that a radio source with a constant jet power, expanding in a medium with a declining density profile, will decrease in radio power (e.g. Baldwin 1982; Kaiser \& Alexander 1997).

\subsection{Perspective from Our Group}

We have used the existing samples of Stanghellini et al. (1998: bright GPS galaxies), Fanti et al. (1990: bright CSS galaxies), and Snellen et al. (1998a: faint GPS galaxies) to study early radio source evolution. This has been described in detail in Snellen et al. (2000). We noticed a distinct difference between the redshift distribution of GPS galaxies from the Stanghellini et al. (1998) sample and 3C galaxies, with the GPS galaxies biased towards higher redshifts (95\% confidence in the KS test). First of all, this makes the number count analyses mentioned above, which are averaged over a large redshift range, less straightforward. Furthermore, it is important to understand how two populations, which evolve into each other on timescales several orders of magnitude less than the Hubble time, can have different redshift distributions. It is clear that to explain this redshift bias in a flux density limited sample, the luminosity function of GPS galaxies has to be different from that of old, extended objects, if the former are to develop into the latter.

We advocate that it is the qualitatively different individual luminosity development of young radio sources compared to that of old objects which causes a flatter overall luminosity function. If radio sources increase in radio power until they grow beyond a certain size and thereafter decrease in radio power, a randomly aged population of compact radio sources will be biased towards higher luminosities than a population of large-size objects, producing a flatter luminosity function. This behaviour in luminosity evolution is expected from theory, in which the density profile of the core of the host galaxy is flat (see Figure 1), and the density only starts to decrease outside this core 


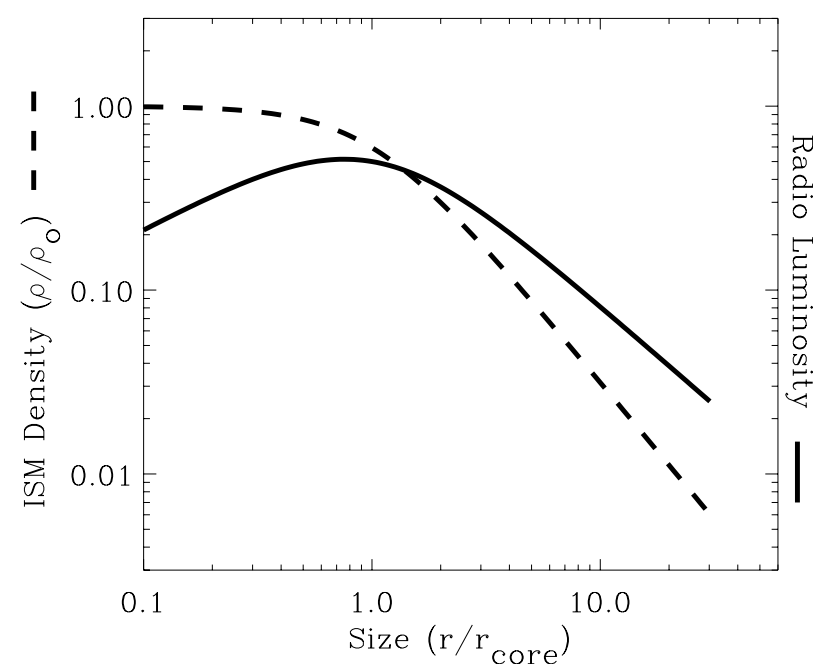

Figure 1 Typical density profile of a galaxy (dashed line), and the expected luminosity evolution as function of size for a radio source (solid line), according to Snellen et al. (2000).

radius (Snellen et al. 2000; Alexander 2000; O’Dea 2003). Some new evidence for this scenario is also presented by Perucho \& Marti (2002, 2003).

\subsection{Synchrotron Self Absorption and Self-Similar Evolution}

Snellen et al. (2000) used the three formerly mentioned samples to study the relation between the radio spectra and the overall sizes of the GPS and CSS sources. When assuming synchrotron self absorption (SSA), the frequency and flux density of the overall spectral turnover provide a good indication of the subtended solid angle of the radio source and subsequently of the size of the dominant radio components, with the angular size, $\theta_{\text {ssa }}$, being

$$
\theta_{\text {ssa }} \propto B^{1 / 4} S_{\text {peak }}^{1 / 2} v_{\text {peak }}^{-5 / 4}
$$

where $B$ is the magnetic field strength. If the weak dependence on $B$ is taken out by assuming an equipartition magnetic field, then the SSA components' sizes are found to be linearly proportional to the overall size of the GPS/CSS sources as measured from VLBI, over a luminosity and linear size range of several orders of magnitude. This indicates that the ratio of overall size to lobe size stays constant while the sources grow, and that therefore young radio sources evolve in a self-similar way. Furthermore, it provides strong evidence that the turnovers in the radio spectra of GPS/CSS sources are indeed caused by SSA, and not free-free absorption (FFA). Further evidence in favour of this scheme is presented by Tschager et al. (2003), who find that a new sample of very faint CSS galaxies also follow the relation given above.

Although it is shown that FFA does occur in some compact GPS sources (e.g. Kameno et al. 2003; Inoue et al. 2003; Vermeulen et al. 2003), it seems that even in these objects FFA only generally affects faint components close to the nucleus, and not the mini-lobes which form the dominant components in the overall radio spectrum. Although it is possible to fit the spectra of individual sources with fine-tuned FFA models, it is not clear to us why the conditions of FFA would change in such a way over a large range of radio source luminosities and linear sizes, that they exactly mimic the frequency and strength of the turnovers as expected for SSA. This makes a very strong case for SSA being the dominant absorption process in GPS and CSS galaxies. Highly accurate flux density monitoring can now be used to further test self-similar evolution by comparing the long-term flux density variability with what is expected for this scenario (e.g. Tingay et al. 2003).

\subsection{Open Questions}

Although we now start to have a more or less general idea of how GPS and CSS sources could evolve into old, extended objects, many questions remain open, and the proposed evolution scenarios should be further tested. Is the initial phase of increase in luminosity correct, and if so, how strong is this rise? Do all GPS/CSS galaxies evolve into extended objects, or is there a significant population of short-lived objects, e.g. due to the lack of fuel (these may show up as dying drop-outs in GPS/CSS samples; e.g. Marecki et al. 2003)? Does intermittance play an important role? Do GPS and CSS sources evolve into FR IIS only, or do they also evolve into FR Is as well? Can we in some way recognise objects which are bound to evolve into either class of object, or are FR Is and IIs physically so closely linked (e.g. through evolution) that there is no difference in their progenitors?

Several other matters have to be settled. The link between the radio spectra and VLBI morphologies has to be further investigated. Do all GPS galaxies have compact symmetric object (CSO) morphologies, such as the bright archetypes? What effect could orientation have on the classification of an object as either a GPS source or a CSO (e.g. Snellen et al. 1998b)? Is Doppler boosting really not important in these objects, as is always assumed, or does it significantly affect our statistical analyses? And even more generally, but not too obviously, how do you exactly decide what is and what is not a GPS source? To address many of these issues, we are studying a new sample of nearby, young radio-loud AGN.

\section{A New, Low-Redshift Sample of Young Radio Sources}

\subsection{Rationale}

For a statistically sound analysis of radio-loud AGN, it is vital to study a complete, volume limited sample, unaffected by possible cosmological evolutionary effects. Evidently, a nearby sample of young radio sources would be the obvious choice, since key observations, such as optical spectroscopy, are much easier to obtain, and the statistics and properties of other (older) classes of radio 
sources are well established at low redshift. Radio source evolution scenarios as proposed above provide distinct predictions on luminosity functions, linear size distributions, and emission line properties and dynamical ages as functions of radio power and size. The determination of these properties will therefore be the main goal of our investigation, which will form a powerful tool to constrain evolution models.

In addition, as a spin-off, a complete sample of nearby young radio-loud AGN allows us to undertake unique studies of their HI absorption distribution (using VLBI in L-band), X-ray, infrared and optical cluster properties. This will undoubtedly give new insights in the intra- and intergalactic environments of young radio-loud AGN.

\subsection{Sample Selection}

Selection of a comprehensive nearby sample has only become possible since the construction of the new generation of radio surveys. In particular, the $1.4 \mathrm{GHz}$ FIRST survey (White et al. 1997) with its high resolution is ideal for selecting compact radio sources, and therefore forms the basis of our sample. Only the region north of declination $+30^{\circ}$ was used, so it overlaps with the WENSS at $325 \mathrm{MHz}$ (Rengelink et al. 1997).

The first step was the selection of all sources in FIRST with an angular size of $<2^{\prime \prime}$ and a flux density $S_{1.4 \mathrm{GHz}}>$ $100 \mathrm{mJy}$. It is important to note that no selection was made on radio spectra, but only on angular size, allowing in a later stage an unbiased investigation of possible orientation and/or beaming effects on the radio spectra and morphologies of young radio-loud AGN. Unfortunately, by selecting at $1.4 \mathrm{GHz}$, sources with spectral turnovers at higher frequencies (very compact objects) may have been missed. To catch these, sources from the flat spectrum CLASS survey (with $S_{5 \mathrm{GHz}}>30 \mathrm{mJy}$; Myers et al. 1995) were added to the sample, with an $8.4-5 \mathrm{GHz}$ spectral index such that their $1.4 \mathrm{GHz}$ flux density would have been brighter than $100 \mathrm{mJy}$, if they were not synchrotron self absorbed.

The positions of the remaining objects were crosscorrelated with the optical APM/POSS-I catalogue, and only those objects which coincided within $10^{\prime \prime}$ with a galaxy with a red magnitude $<16.5$ were selected. This yielded a total of 55 objects, which were subsequently correlated with other surveys such as 4C, NVSS (Condon et al. 1998), GB6 (Gregory et al. 1996), and CLASS, and images were obtained from the NVSS and the Digitized Sky Survey (DSS: Lasker et al. 1990) to check whether the optical identification was genuine, and to see whether any large scale radio emission may have been missed (on which basis they would be rejected). The literature was searched for any available redshifts, and optical spectra were taken for the remaining objects with the Calar Alto $2.2 \mathrm{~m}$ and the $2.5 \mathrm{~m}$ Isaac Newton telescopes.

The final sample contains 28 compact radio sources in the redshift range $0.008<0.232$. The redshift distribution is shown in Figure 2.

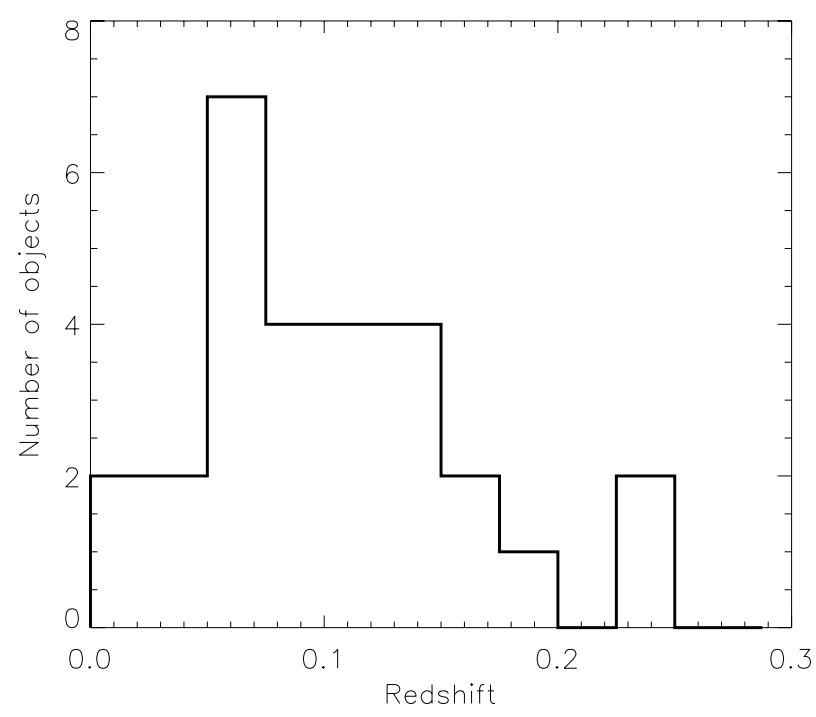

Figure 2 The redshift distribution of the sources in the new sample of nearby young radio-loud AGN.

\subsection{First Observations and Results}

A comprehensive observational program is underway to study the individual objects in detail. VLA observations at $5,8.4$, and $15 \mathrm{GHz}$, and Effelsberg observations at $2.7,4.8,10.5$, and $32 \mathrm{GHz}$ were taken to refine the radio spectra and to determine possible flux density variability. The shapes of the radio spectra have given us a first indication of the angular sizes expected for these objects (see Section 1.2). This information was used to divide the sample into small-size, intermediate-size, and largesize sub-samples. These sub-samples were then observed with the VLBA+EVN at $5 \mathrm{GHz}(\sim 1.5$ mas resolution), the EVN at $1.6 \mathrm{GHz}(\sim 15$ mas resolution), and MERLIN at $1.6 \mathrm{GHz}(\sim 150$ mas resolution) respectively. So far, all but one source have been observed in this way. In addition, optical imaging in B, V, R, and I band filters, and HI absorption observations with WSRT are underway.

The first results look very promising. More than $80 \%$ of the objects have radio spectra which can be classified as classical GPS or CSS sources (see Figure 3), with the remainder also showing a spectral peak but clearly exhibiting variability. Most of the objects have morphologies consistent with them being compact double or compact symmetric objects, as expected for this class of objects, but some seem to show core-jet or more complex structures. This sample is ideal to further study the relation between radio spectrum, morphology, orientation, and Doppler boosting.

At this stage, the foundation has been laid to determine directly the local luminosity function, source size distribution, and the dynamical age distribution for young radio-loud AGN, without cosmological bias.

\section{Acknowledgements}

We wish to thank Tasso Tzioumis for the excellent organisation of the workshop, and the villagers of Kerastari for 


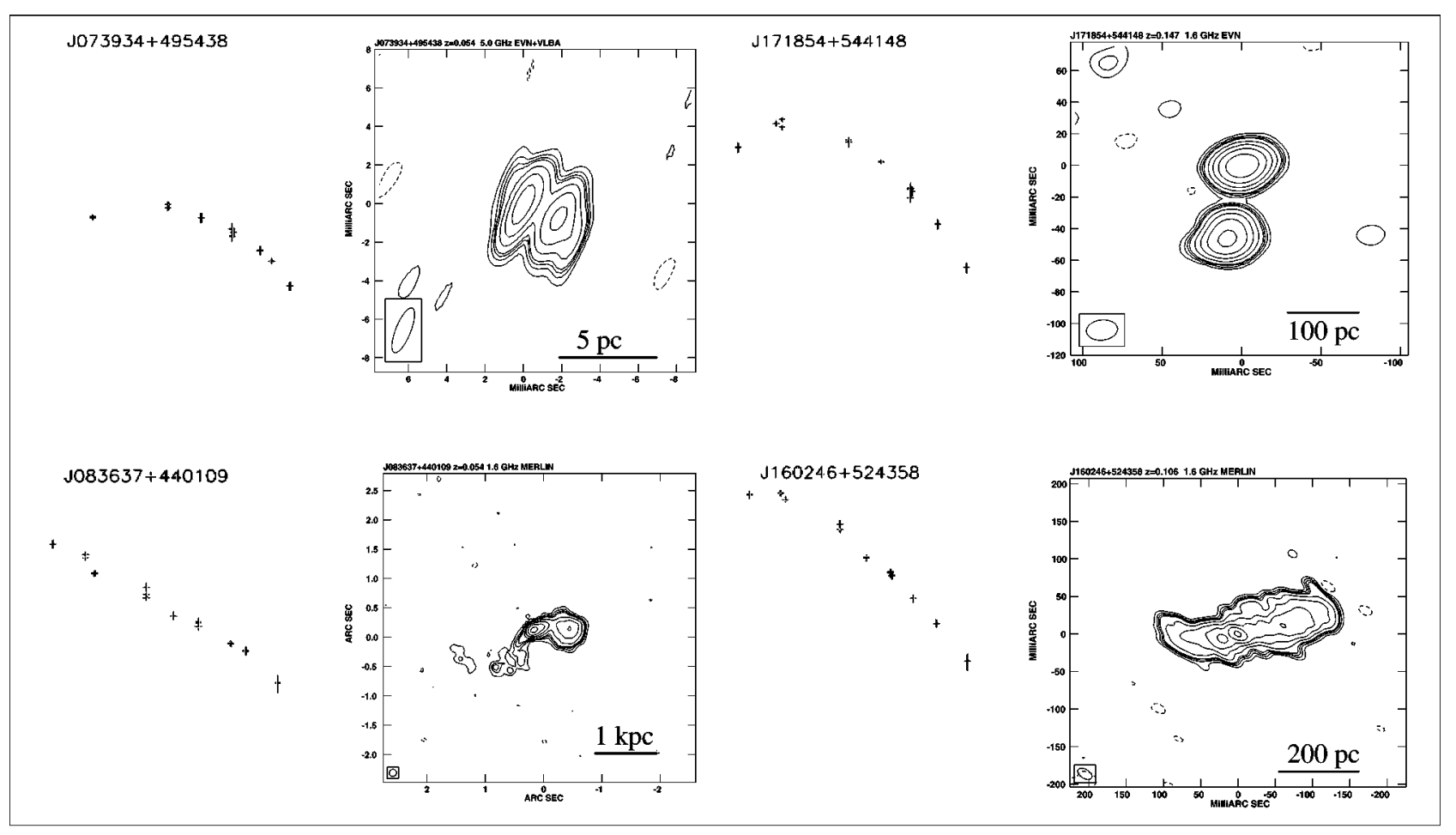

Figure 3 Example radio spectra and morphologies of sources in the new sample of nearby young radio-loud AGN.

their warm hospitality. This paper is partly based on observations collected at the German-Spanish Astronomical Center, Calar Alto, Spain, operated by the Max-PlanckInstitute für Astronomie, Heidelberg, jointly with the Spanish National Commission for Astronomy; the Isaac Newton Telescope (through the service program), which is operated on the island of La Palma by the Isaac Newton Group in the Spanish Observatorio del Roque de los Muchachos of the Instituto de Astrofisica de Canarias; the European VLBI Network (EVN, making use of EC's Access to Research Infrastructures Program, under Contract No. HPRI-CT-1999-00045), which is a joint facility of European, Chinese and other radio astronomy institutes funded by their national research councils; MERLIN, a National Facility operated by the University of Manchester at Jodrell Bank Observatory on behalf of PPARC. The National Radio Astronomy Observatory is a facility of the National Science Foundation operated under cooperative agreement by Associated Universities, Inc. KHM was supported by a Marie Curie fellowship of the European Commission.

\section{References}

Alexander, P. 2000, MNRAS, 319, 8

Baldwin, J. 1982, in Extragalactic Radio Sources, eds D. S. Heeschen \& C. M. Wade (Dordrecht: Reidel), 21

Condon, J. J., Cotton, W. D., Greisen, E. W., Yin, Q. F., Perley, R. A., Taylor, G. B., \& Broderick, J. J. 1998, AJ, 115, 1693

Fanti, R., Fanti, C., Schilizzi, R. T., Spencer, R. E., Nan Rendong, Parma, P., van Breugel, W. J. M., \& Venturi, T. 1990, A\&A, 231,333
Fanti, C., Fanti, R., Dallacasa, D., Schilizzi, R. T., Spencer, R. E., \& Stanghellini, C. 1995, A\&A, 302, 317

Gregory, P. C., Scott, W. K., Douglas, K., \& Condon, J. J. 1996, AJS, 103,427

Inoue, et al. 2003, PASA, submitted

Kaiser, C. R., \& Alexander, P. 1997, MNRAS, 286, 215

Kameno, et al. 2003, PASA, submitted

Lasker, B. M., Sturch, C. R., McLean, B. J., Russell, J. L., Jenkner, H., \& Shara, M. M., 1990, AJ, 99, 2019

Marecki, et al. 2003, PASA, in press

Myers, S. T., et al. 1995, AJ, 447, L5

O’Dea, C. P., \& Baum, S. A. 1997, AJ, 113, 148

O'Dea, 2003, PASA, submitted

Perucho, M., \& Marti, J. M. 2002, ApJ, 568, 639

Perucho, M., \& Marti, J. M. 2003, PASA, in press

Polatidis, A., \& Conway, J. 2003, PASA, 20, in press

Readhead, A. C. S., Taylor, G. B., Xu, W., Pearson, T. J., \& Wilkinson, P. N. 1996, ApJ, 460, 634

Rengelink, R. B., Tang, Y., de Bruyn, A. G., Miley, G. K., Bremer, M. N., Röttgering, H. J. A., \& Bremer, M. A. R. 1997, A\&AS, 124, 259

Snellen, I. A. G., Schilizzi, R. T., de Bruyn, A. G., Miley, G. K., Rengelink, R. B., Röttgering, H. J. A., \& Bremer, M. N. 1998a, A\&AS, 131, 435

Snellen, I. A. G., Schilizzi, R. T., de Bruyn, A. G., \& Miley, G. K. 1998b, A\&A, 333, 70

Snellen, I. A. G., Schilizzi, R. T., Miley, G. K., de Bruyn, A. G., Bremer, M. N., \& Röttgering, H. J. A. 2000, MNRAS, 319, 445

Stanghellini, C., O’Dea, C. P., Dallacasa, D., Baum, S. A., Fanti, R., \& Fanti, C. 1998, A\&AS, 131, 303

Tingay, S., et al. 2003, PASA, in press

Tschager, et al. 2003, PASA, submitted

Vermeulen, R. C., Ros, E., Kellermann, K. I., Cohen, M. H., Zensus, J. A., \& van Langevelde, H. J. 2003, PASA, 20, in press

White, R. L., Becker, R. H., Helfand, D. J., \& Gregg, M. D. 1997, ApJ, 475, 479 